Research Paper

\title{
Circular RNA hsa-circ-0007766 modulates the progression of Gastric Carcinoma via miR-1233-3p/GDFI5 axis
}

\author{
Weiguo $\mathrm{Xu}^{1 \#}$, Bin Zhou ${ }^{1 \#}$, Jun $\mathrm{Wu}^{2}$, Pan Jiang ${ }^{3}$, Huanqiu Chen ${ }^{1 凶}$ and Feng Yan ${ }^{3 凶}$ \\ 1. Department of General Surgery, The Affiliated Cancer Hospital of Nanjing Medical University \& Jiangsu Cancer Hospital \& Jiangsu Institute of Cancer \\ Research, Nanjing 210009, China. \\ 2. Department of Clinical Laboratory, The Affiliated Brain Hospital of Nanjing Medical University, Nanjing 210009, China. \\ 3. Department of Clinical Laboratory, The Affiliated Cancer Hospital of Nanjing Medical University \& Jiangsu Cancer Hospital \& Jiangsu Institute of Cancer \\ Research, Nanjing 210009, China.
}

\#These authors contributed equally to this work.

$\triangle$ Corresponding authors: Dr. Feng Yan. Department of Clinical Laboratory, The Affiliated Cancer Hospital of Nanjing Medical University \& Jiangsu Cancer Hospital \& Jiangsu Institute of Cancer Research, Baiziting No.42, Nanjing 210009, Jiangsu, China. Tel.: +86-25-8328-3401; Fax: +86-25-8328-3312; E-mail: yanfeng@jszlyy.com.cn; Dr. Huanqiu Chen. Department of General Surgery, The Affiliated Cancer Hospital of Nanjing Medical University \& Jiangsu Cancer Hospital \& Jiangsu Institute of Cancer Research, Baiziting No.42, Nanjing 210009, Jiangsu, China. Tel.: +86-25-8328-3341; Fax: +86-25-8328-3341; E-mail: chenhuanqiu@jszlyy.com.cn.

(C) The author(s). This is an open access article distributed under the terms of the Creative Commons Attribution License (https://creativecommons.org/licenses/by/4.0/). See http://ivyspring.com/terms for full terms and conditions.

Received: 2020.03.23; Accepted: 2020.06.12; Published: 2020.06.23

\begin{abstract}
Circular RNAs (circRNAs), a new kind of non-coding RNAs, have gradually been proved to be critical regulators of gene expression; however, the underlying mechanisms still need to be elaborated. In the present study, we investigated the role of hsa-circ-0007766 in gastric carcinoma (GC). Quantitative real-time PCR was applied to detect the differential expression levels of circRNA, miRNAs, and mRNAs in human tissues and specific cell lines. GC cell lines were transiently transfected with siRNA. Then the proliferation, migration, and invasion assays were performed to evaluate the effect of hsa-circ-0007766 in GC cell lines. Fluorescence in situ hybridization, RNA pulldown assay was used to confirm the location of hsa-circ-0007766 and its relationship with miR-1233-3p. Luciferase reporter assay was then conducted to verify the interaction between miR-1233-3p and GDFI5. Interestingly, we found that hsa-circ-0007766 was highly expressed in human GC tissues and GC cell lines. Knock-down of hsa-circ-0007766 inhibited cell proliferation, migration, invasion, and down-regulated the expression of GDFI5. Moreover, hsa-circ-0007766 was identified as a sponge of miR-1233-3p, which could target gene GDF15 to regulate the progression of GC. Finally, hsa-circ- 0007766 was evaluated to be a valuable diagnostic marker with a sensitivity of $53.33 \%$ and specificity of $83.33 \%$ by ROC analysis. This study unveils a mechanism by which hsa-circ-0007766 regulates GDFI5 via hsa-circ-0007766/miR-1233-3p/GDFI5 axis, which may provide new insight for GC therapeutic strategies.
\end{abstract}

Key words: hsa-circ-0007766, miR-1233-3p, GDF15, gastric carcinoma

\section{Introduction}

Gastric cancer is a common malignant tumor in the world, and its prognosis is relatively poor [1]. According to the statistics of The International Agency for Research on Cancer (IARC) in 2012, there were about 951,000 new cases of gastric cancer in the world and about 723,000 deaths due to gastric cancer, which was ranked 5th in the incidence of malignant tumors and 3rd in mortality respectively [2]. Despite significant advances in the field of individualized therapy, gastric cancer (GC) remains a clinically challenging disease due to the lack of reliable molecular tools to predict the outcomes of patients. Therefore, a better understanding of the molecular mechanisms involved in the progression of gastric cancer is significant for the development of new therapeutic strategies [3]. 
Circular RNAs (circRNAs), circular closed-loop RNA molecules that competitively inhibit target genes by absorbing specific miRNAs, regulate cell proliferation, differentiation, and apoptosis, and participate in tumorigenesis [4-8]. They play an essential role in the growth and development of human diseases such as malignant tumors of the digestive tract, heart diseases, nervous system diseases, and diabetes [9-11]. Due to its high plasma concentration, and high specificity, circRNAs have the potential to become a novel biomarker and play a vital role in the early diagnosis, treatment, and prognosis of tumors [11, 12]. Besides, the recent evidence suggests that circRNAs have the function of translating proteins, which indicates that circRNAs are no longer strictly non-coding RNAs and provides a new direction for studying the role of circRNAs in tumors [7, 13]. We experimentally searched the CircBase [14], CircInteractome [15], Starbase v2.0 [16], and found that hsa-circ-0007766 is generated from five exons $(676 \mathrm{bp})$ of erb-b2 receptor tyrosine kinase 2 (ERBB2, chr17:37864573-37866734). We then selected hsa-circ-0007766 as the target of our research as the emerging application of Trastuzumab in Her2 (ERBB2) positive patients diagnosed with gastric carcinoma [17]. To our knowledge, it is the first time to elaborate on the biological characteristics of hsa-circ-0007766 in GC.

Growth Differentiation Factor 15 (GDF15), a member of transforming growth factor-beta superfamily, has been used to predict disease progression in cancer, cardiovascular disease, chronic renal dysfunction, and pulmonary embolism [18-23]. Also, GDF15 was accepted as a factor to promote cell viability, invasion, migration, angiogenesis, and apoptosis in human GC cell lines [24, 25]. MicroRNAs (miRNAs) are a class of single-stranded, endogenous, non-coding RNAs that play an essential role in regulating the expression of specific genes by binding to the 3'UTR of mRNA [26]. As circRNAs were reported to be miRNAs sponge, we speculated that GDF15 could be regulated by sponge effect. Therefore, we assumed that hsa-circ-0007766 might be involved in the progression of GC by regulating the expression of GDF15 via the miR-1233-3p/GDF15 axis.

\section{Materials and Methods}

\section{Tissue samples}

Totally 30 pairs of GC samples were obtained from patients diagnosed with GC who underwent gastrectomy in The Affiliated Cancer Hospital of Nanjing Medical University between 2015 and 2017. All samples were snap-frozen and stored at $-80{ }^{\circ} \mathrm{C}$ until RNA or protein extraction. The study was conducted following the Declaration of Helsinki. These patients signed all informed consent, and the ethics committee has approved this study of The Affiliated Cancer Hospital of Nanjing Medical University (NYDLS-2019-919).

\section{Cell culture and transfection}

Human gastric cancer cell lines SNU-216, HGC27, BGC-823, SGC-7901, and the human gastric epithelial cell line GES-1 were obtained from the Type Culture Collection of the Chinese Academy of Sciences (Shanghai, China). SNU-216 and GES-1 were cultured with DMEM medium (Gibco, USA) containing $10 \%$ fetal bovine serum (FBS). BGC-823 and SGC-7901 were cultured with RPMI 1640 medium (Gibco, USA) with 10\% FBS. HGC-27 was cultured with RPMI 1640 medium (Gibco, USA) with 20\% FBS. The cell lines were cultured in a humidified incubator at $37{ }^{\circ} \mathrm{C}$ with $5 \% \mathrm{CO}_{2}$. The cells were harvested by trypsinization and seeded in a 6-well plate $\left(2 \times 10^{5}\right.$ cells/well). Twenty-four hours later, $100 \mathrm{nM}$ of siRNAs or miRNAs were transfected into cells using Lipofectamine 2000 (Invitrogen, USA). The sequences of the siRNA and control group were demonstrated in Table 1. The detailed procedure of transfection was conducted according to the manufacturer's instructions.

\section{RNA extraction and quantitative real-time polymerase chain reaction (qRT-PCR)}

Total RNA was extracted with TRIzol reagent (Invitrogen, USA), and cDNA was synthesized with the PrimeScript RT reagent Kit (Takara, Japan) or the Bulge-Loop miRNA Starter Kit (RiboBio, Guangzhou, China) according to the instructions. Then, qPCR was performed with SYBR Premix Ex Taq (Takara, Japan) in LightCycler 1.5 (Roche, Switzerland). PCR amplification was performed in a $20 \mu \mathrm{L}$ reaction system including $2 \mu \mathrm{L}$ cDNA, $6 \mu \mathrm{L}$ DEPC, $10 \mu \mathrm{L}$ SYBR Green Mix, $1 \mu \mathrm{L}$ forward primer, and $1 \mu \mathrm{L}$ reverse primer. RT reaction conditions were $60 \mathrm{~min}$ at $42{ }^{\circ} \mathrm{C}$ and $10 \mathrm{~min}$ at $70^{\circ} \mathrm{C}$. PCR conditions were as follows: $95^{\circ} \mathrm{C}$ for $2 \mathrm{~min}$; 40 cycles of $95^{\circ} \mathrm{C}$ for $15 \mathrm{sec}$ and $60^{\circ} \mathrm{C}$ for $60 \mathrm{sec}$. GAPDH served as an internal control for hsa-circ-0007766 and GDF15, and U6 was used as an internal control for miRNAs. The primers used in qPCR were listed in Table 1. Total RNA was incubated for $15 \mathrm{~min}$ at $37^{\circ} \mathrm{C}$ with 3 units of RNase R (Epicentre) per $1 \mathrm{mg}$ RNA. RNA was subsequently purified by phenol-chloroform extraction and reprecipitated in three volumes of ethanol. The relative expression levels were calculated using the $2^{-\Delta \Delta \mathrm{Ct}}$ method. All results were expressed as mean \pm SEM of three independent experiments. 


\section{Cell proliferation assay and colony formation assay}

About 2000 transfected cells were seeded into each well of 96 -well plates, incubated at $37^{\circ} \mathrm{C}$ with $5 \%$ CO2. CCK-8 solution $(10 \mu \mathrm{L})$ was added to each well, and absorbance at $450 \mathrm{~nm}$ was detected at $0,24,48,72$, 96 hours after seeding with a microplate reader. Then, about 1000 transfected cells were seeded in each well of 6-well plates and cultured for ten days. Cell colonies were fixed using $4 \%$ paraformaldehyde for $20 \mathrm{~min}$ and stained with Giemsa solutions (KeyGen Biotech, China) for another $20 \mathrm{~min}$. Colony numbers in each group were counted using ImageJ software.

\section{Migration and invasion assays}

In this study, about $5 \times 10^{4}$ transfected cells were suspended in $200 \mu \mathrm{L}$ serum-free medium and seeded into the upper chamber without Matrigel for migration assay. Analogously, about $8 \times 10^{4}$ transfected cells containing $200 \mu \mathrm{L}$ serum-free medium were seeded into the Transwell Matrigelcoated upper chamber (Corning ${ }^{\circledR}$ Matrige ${ }^{\circledR}$ invasion chamber, USA) for invasion assay. Medium with 10\% FBS was added into the lower chamber as a chemoattractant. The cells were incubated for 24 hours for migration assay and 48 hours for invasion assay. We removed the cells on the top side of the chamber with cotton swabs. Then the bottoms of the chambers were fixed with $4 \%$ paraformaldehyde for $20 \mathrm{~min}$ and stained with $0.1 \%$ crystal violet for another $20 \mathrm{~min}$. Finally, we counted the invaded cells and captured the photographs with a microscope (magnification $\times 100$ ).

\section{CircRNA Fluorescent in situ Hybridization (FISH)}

Cy3-labeled hsa-circ-0007766 probe and negative control were purchased from RiboBio (Guangzhou, China). A Ribo ${ }^{\mathrm{TM}}$ ncRNA FISH kit (cat. no. C10910) purchased from RiboBio (Guangzhou, China) was employed for circRNA FISH to identify the location and expression of hsa-circ-0007766 in gastric cancer cell lines (HGC-27 and SNU-216) according to the manufacturer's protocol. In brief, cells were cultured and fixed in $4 \%$ paraformaldehyde for $10 \mathrm{~min}$ at room temperature. Cells were permeabilized with Triton-100 for $5 \mathrm{~min}$ at $4{ }^{\circ} \mathrm{C}$ (Beyotime, Shanghai, China), then Cy3-labelled hsa-circ-0007766 and DAPIlabelled 18S-RNA probes (RiboBio, Guangzhou, China) were detected. In the dark, DNA was stained with DAPI for $10 \mathrm{~min}$ at room temperature, followed by washing in PBS three times every $5 \mathrm{~min}$. Slides were mounted and examined by confocal fluorescence microscopy $(\times 200$; Olympus Corporation, Tokyo, Japan).

\section{Pulldown assay with Biotin-labelled circRNA probe}

The biotinylated probe was synthesized by RiboBio (Guangzhou, China). The sequence of the probe was specifically designed to bind to the back-spliced junction of hsa-circ-0007766, while the scramble probe was used as the negative control. About $1 \times 10^{7}$ cells were lysed in lysis buffer $(25 \mathrm{mM}$ Tris $\bullet \mathrm{HCl} \mathrm{pH} 7.4,150 \mathrm{mM} \mathrm{NaCl}, 6 \mathrm{mM} \mathrm{MgCl}$, $1 \%$ NP-40, $1 \mathrm{mM}$ EDTA, 5\% glycerol, $1 \times$ Proteinase Inhibitor cocktail, $60 \mathrm{U} / \mathrm{mL}$ RNase inhibitor). Cell lysates were incubated with $3 \mu \mathrm{g}$ biotinylated probes or scramble probes for two hours in RT. Then the biotin-circRNA-RNA complex was pulled down by incubating the lysates with washed streptavidin magnetic beads (Invitrogen, USA) for another four hours in $4{ }^{\circ} \mathrm{C}$. Finally, the beads were washed five times with lysis buffer, and RNA complexes combining on the beads were extracted with TRIzol reagent for further research.

\section{Pulldown assay with Biotin-labelled miRNA probe}

The biotinylated miRNA mimics probe and the scramble probe were designed by RiboBio (Guangzhou, China). The sequences of probes were listed in Table 1 . About $2 \times 10^{6}$ cells were transfected with $50 \mu \mathrm{M}$ of biotinylated miRNA mimics probe or the scramble probe at $50 \%$ confluency for lysis. The lysates were incubated with blocked streptavidin magnetic beads (Invitrogen, USA) for four hours in 4 ${ }^{\circ} \mathrm{C}$. Next, the beads were washed five times, and the binding RNAs were purified with TRIzol reagent for qRT-PCR. Then we analyzed the product of PCR by agarose gel electrophoresis.

\section{Dual-luciferase reporter assay}

GC cell lines HGC-27 and SNU-216 were cultured in 24-well plates and then cells were cotransfected with plasmids containing 3'UTR of GDF15 and miR-1233-3p mimics using Lipofectamine 2000 (Invitrogen, USA). The GDF15 3'-UTR wild-type and a GDF15 3'-mutant were synthesized and inserted into the pmirGLO luciferase reporter vector to produce pmirGLO-GDF15-wt and pmirGLO-GDF15-mut constructs, respectively. The cells were co-transfected with wild-type or mutant-type reporter plasmid vectors and miR-1233-3p mimics or negative control. The luciferase activities of firefly and renilla were detected by the dual-luciferase reporter assay system (Promega, Massachusetts, USA). The ratios were calculated by normalizing the firefly luciferase activity with that of renilla, and each test was repeated for three independent assays. 
Table 1. The sequence of primers and probes

\begin{tabular}{ll}
\hline Names & Sequence $\left(5^{\prime}\right.$ - $\left.^{\prime}\right)$ \\
\hline hsa-circ-0007766-F & TGTGTGACTGCCTGTCCCTG \\
hsa-circ-0007766-R & AATCCGCAGCCTCTGCAGTG \\
si-hsa-circ-0007766 (si-circ) & TGCCTGTCCCTGATATCCA \\
GDF15-F & TGCCTGTCCCTGATATCCA \\
GDF15-R & GCAGGTCCTCGTAGCGTTTC \\
U6-F & CTCGCTTCGGCAGCACA \\
U6-R & AACGCTTCACGAATTTGCGT \\
hsa-circ-0007766-biotin-probe & ATATCAGGGACAGGCAGTCA \\
hsa-circ-0007766-scramble-probe & ATGACTGAGTCGCGAAGCAA \\
gDNA-F & TCACAGAGATCTTGAAAGGAGGG \\
gDNA-R & GAGAGCGGTTGGTGTCTATCAG \\
GAPDH-F & ACCCACTCCTCCACCTTTGAC \\
GAPDH-R & TGTTGCTGTAGCCAAATTCGTT \\
\hline
\end{tabular}

Note F, forward; R, reverse. The sequence information of all siRNA controls, ASO controls, miRNA primers, and Smart Silencer controls is not public according to the declaration of RiboBio (Guangzhou, China).

\section{Western blot}

We used RIPA lysis buffer (Beyotime, Shanghai, China) to extract protein from SNU-216 and HGC-27 cells. Then BCA Protein assay kit (Beyotime, Shanghai, China) was applied to detect the protein concentration. Protein extractions $(100 \mu \mathrm{g})$ were separated by $10 \%$ SDS-PAGE and transferred onto polyvinylidene fluoride (PVDF) membranes. Afterwards, the membrane was blocked with 5\% nonfat milk (BD Biosciences, USA) and 0.1\% Tween 20 in tris-buffered saline. Then the blocked membrane was immunoblotted overnight using anti-GDF15 antibody (1:1000, \#A0185; ABclonal, China) and antibeta-actin antibody (1:2000, \#AC026; ABclonal, China) at $4{ }^{\circ} \mathrm{C}$ with gentle shaking. The membranes were blocked in secondary antibodies (1:5000, ABclonal, China) at room temperature for two hours, and protein bands were visualized by an ECL kit (Keygen, Nanjing, China). Band intensity was quantified using a chemiluminescent and multicolor fluorescent imaging system and normalized to the respective Actin bands. Immunoreactivity was detected with the Odyssey fluorescent scanning system (LI-COR) and analyzed by Image Studio software.

\section{Statistical analysis}

All the analyses were performed with SPSS 19.0 (IBM, SPSS, Chicago, IL, USA) and GraphPad Prism 6, and all data were presented as mean \pm standard error of the mean (SEM). Significant differences were calculated using the Student's t-test or Chi-square test. Pearson's Coefficient was conducted to analyze the pertinence of qPCR results. Software MedCalc was applied to make the receiver operating characteristic (ROC) curve and calculate the area under curve (AUC), sensitivity, and specificity. The sensitivityspecificity relationship was determined using the Youden's J index. $\mathrm{P}<0.05$ was considered to be statistically significant.

\section{Results}

\section{Hsa-circ-0007766 was high-expressed in GC tissues and cell lines and was correlated with the clinical-pathological parameters}

Hsa-circ-0007766 was selected by us with several databases, and it was validated by RNase $\mathrm{R}$ digestion and qRT-PCR (Figure 1). We observed that hsa-circ0007766 was resistant to RNase R, whereas the linear control was not stable with RNase R treatment (Figure $2 \mathrm{~A})$. We then designed the divergent primers for hsa-circ-0007766 and verified the qRT-PCR product with Sanger sequencing (Figure 2B-2C). Next, the divergent and convergent primers for hsa-circ0007766 were designed to amplify the cDNA of circRNA and gDNA of linear RNA from GC cell line HGC-27 by qRT-PCR. We confirmed that cDNA of circRNA could be amplified by both primers; however, gDNA could only be amplified by convergent primers. The results of Sanger sequencing verified the back-spliced junction, and the gel electrophoresis was applied to confirm the product of qPCR for circRNA and linear RNA (Figure 2D-2E). Then we detected 30 pairs of GC tissues and matched adjacent normal tissues with qRT-PCR to verify the expression level of hsa-circ-0007766. We found hsacirc-0007766 was significantly high-expressed in these tissues and several GC cell lines (SNU-216, HGC-27, BGC-823, SGC-7901) compared with matched adjacent normal tissues and normal gastric epithelial cell line GES-1, respectively (Figure 2F-2G). And designed siRNA could efficiently down-regulate the expression of hsa-circ-0007766 (Figure 2H). Besides, we analyzed the results of qRT-PCR, we found that the expression level of hsa-circ-0007766 was significantly correlated with histological grade rather than other parameters (age, sex, tumor size, TNM stage) (Table 2). We then conducted the FISH assay in GC cell lines and found hsa-circ-0007766 was mainly located in the cell cytoplasm (Figure 2I).

\section{Down-regulated hsa-circ-0007766 inhibited the progression of $\mathbf{G C}$ cells in vitro}

SiRNA or negative control (NC) was transiently transfected into SNU-216 or HGC-27 with lipofectamine 2000 to investigate the underlying mechanism of hsa-circ-0007766 in GC cell lines. We found that the expression level of hsa-circ-0007766 was down-regulated for about 10-16 folds with siRNA (Figure 2H). The CCK-8 assay suggested that downregulated circRNA could inhibit the proliferation of SNU-216 and HGC-27 cell lines compared with the NC group obviously (Figure 3A-3B). Besides, transwell migration and invasion assays demonstrated that knockdown of hsa-circ-0007766 
could also suppress the migration and invasion abilities of SNU-216 and HGC-27 cell lines (Figure 3C-3F). We then applied colony formation assays to assess the growth ability of GC cells and found that down-regulated hsa-circ-0007766 could significantly suppress the growth of SNU-216 and HGC-27 cell lines (Figure 3G-3J). The assays above showed that down-regulated hsa-circ-0007766 inhibited the progression of GC cells in vitro.

\section{Hsa-circ-0007766 acted as the miRNA sponge for miR-1233-3p}

To explore the downstream miRNAs, we analyzed CircInteractome [15], Starbase v2.0 [16], RNAhybrid [27] and TargetScan [28] to predict the binding sites of hsa-circ-0007766 with miR-1233-3p (Figure 1, Figure 4A). We found that hsa-circ-000776 might probably bind with several miRNAs (miR1233-3p, miR-1276, miR-136-5p, miR-187, miR-566, miR-637, miR-34a, miR-449a, miR-449b-5p, miR-377$3 p$, miR-34a-5p, miR-34c-5p). We attempted to figure out the expression correlation between hsa-circ0007766 and these miRNAs by qRT-PCR, and we found that miR-1233-3p was significantly upregulated after the knockdown of hsa-circ-0007766 (Figure 4B). Consistently, a pulldown assay with a biotin-labelled circRNA/miRNA probe further confirmed this interaction. As shown in Figure 4C, miR-1233-3p pulled down by biotin-labelled hsacirc-0007766 could be detected by qRT-PCR compared with the scramble group. Meanwhile, biotin-labelled miR-1233-3p captured more hsa-circ-0007766 than the biotin-NC (Figure 4D-4E). These data confirmed the interaction between hsa-circ-0007766 and miR-1233$3 p$. Then we demonstrated the correlation between miR-1233-3p levels and the prognostic outcomes of
GC patients using the Kaplan-Meier Plotter website [29]. Patients were stratified into two groups based on an auto select best cut-off. We found that miR-1233-3p expression levels were correlated with survival data (Figure 4F).

\section{GDF 15 was a downstream target of miR-1233-3p}

We consulted related literature to find that GDF15 might be the downstream gene of miR-1233-3p (Figure 1, Figure 5A). Then luciferase reporter assay was conducted to verify this binding between GDF15 mRNA ( $3^{\prime}$ UTR of wild or mutant fragments) and miR-1233-3p, and we found that miR-1233-3p mimics could significantly reduce the ratios of luciferase activity (Figure 5B-5C). MiR-1233-3p mimics could significantly up-regulate the expression of miR-1233$3 p$ in SNU-216 and HGC-27 (Figure 5D). Next, miR1233-3p mimics were transfected into GC cell lines, and we found that the expression of GDF15 was significantly reduced by qRT-PCR or western blot (Figure 5E-5F).

Table 2. Correlations between the expression level of hsa-circ-0007766 and the clinicopathological features in 30 pairs of $\mathrm{GC}$ tissues

\begin{tabular}{|c|c|c|c|c|c|}
\hline \multicolumn{2}{|c|}{ Characteristics } & \multirow[t]{2}{*}{ Case } & \multicolumn{2}{|c|}{ Circ-0007766 expression } & \multirow[t]{2}{*}{$\mathrm{p}$-value } \\
\hline & & & Low & High & \\
\hline$\overline{\text { All }}$ & & 30 & 17 & 13 & \\
\hline \multirow{2}{*}{ Age (years) } & $<65$ & 11 & 7 & 4 & 0.708 \\
\hline & $\geq 65$ & 19 & 10 & 9 & \\
\hline \multirow[t]{2}{*}{ Gender } & Male & 17 & 12 & 5 & 0.196 \\
\hline & Female & 13 & 12 & 1 & \\
\hline \multirow[t]{2}{*}{ Histology } & Poor-differentiated & 15 & 5 & 10 & $0.025^{*}$ \\
\hline & well-differentiated & 15 & 12 & 3 & \\
\hline \multirow[t]{2}{*}{ TNM stage } & I-II & 5 & 2 & 3 & 0.628 \\
\hline & III-IV & 25 & 15 & 10 & \\
\hline
\end{tabular}

${ }^{*} \mathrm{p}<0.05$.

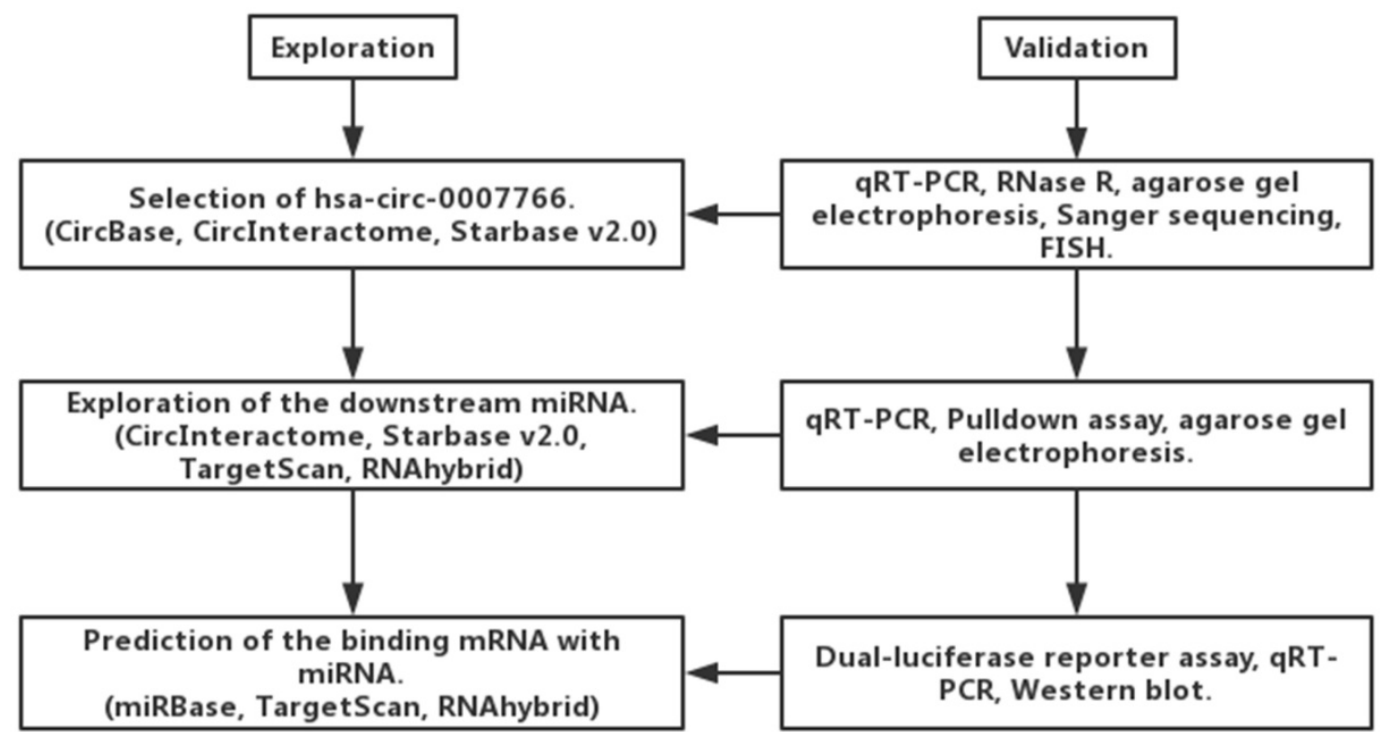

Figure 1. Exploration and validation of circRNA, miRNA, and mRNA based on the bioinformatic analysis. 
A

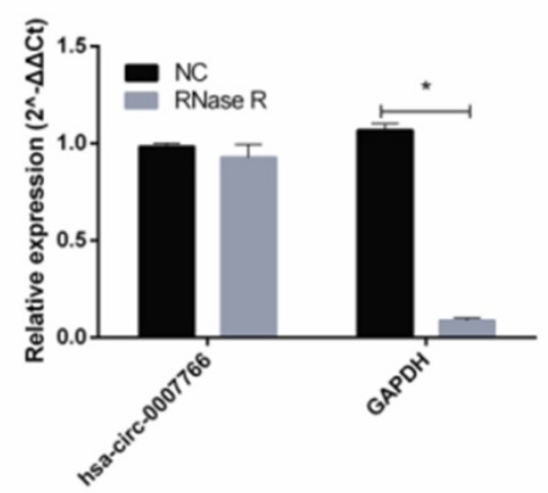

D

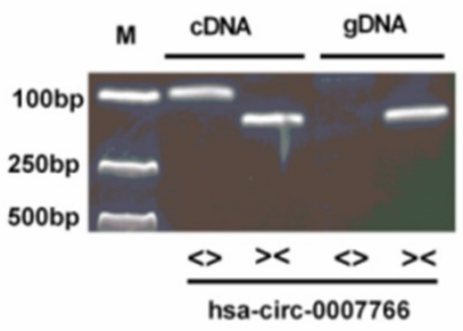

G

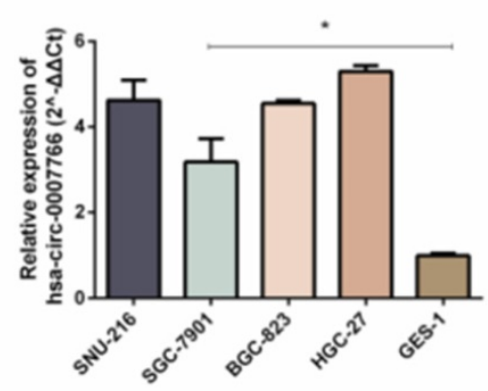

I
B

$97 \mathrm{bp}$

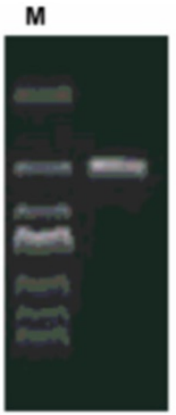

E

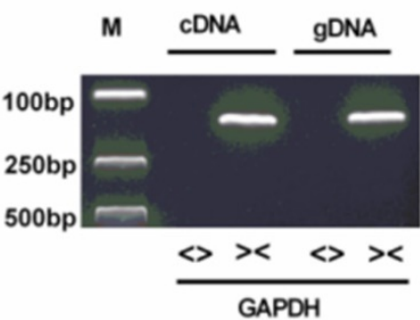

C

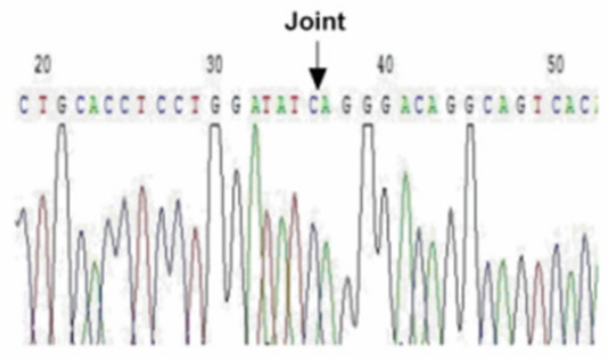

$\mathbf{F}$

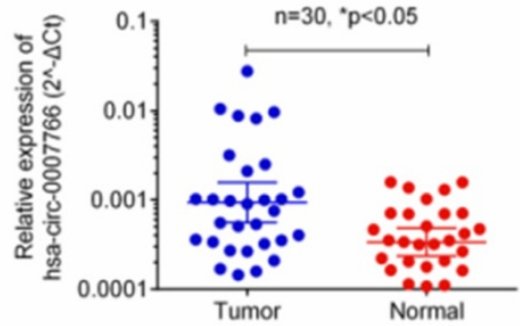

H

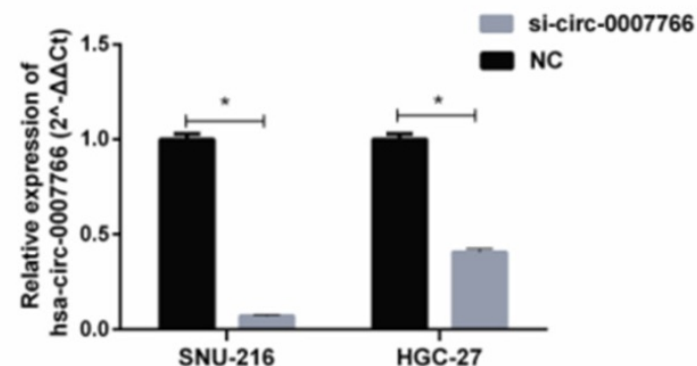

DAPI

Merge
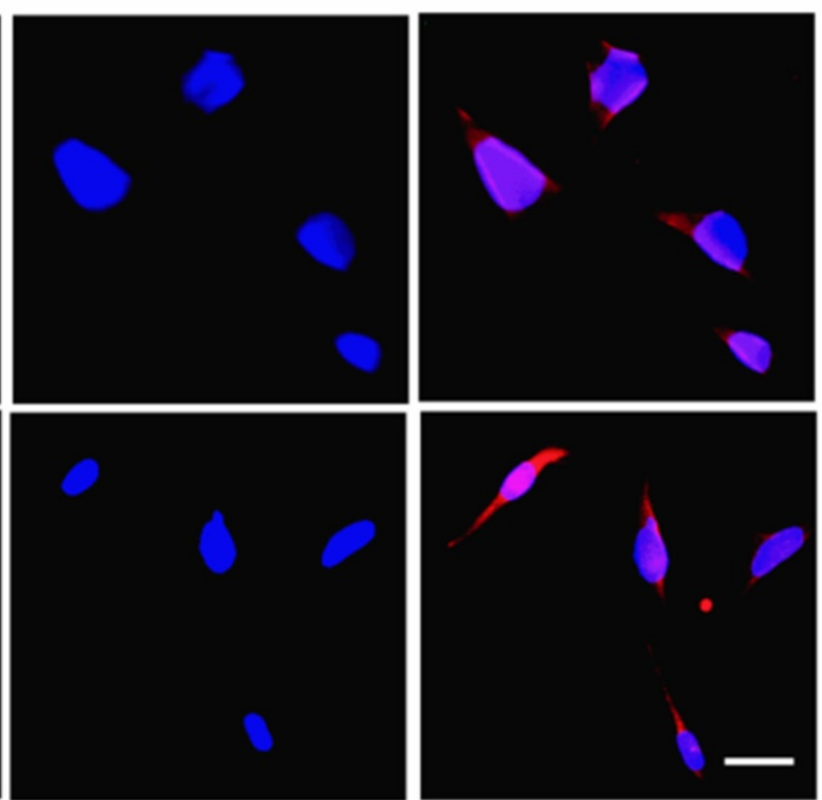

Figure 2. Hsa-circ-0007766 were verified and high-expressed in GC tissues and cell lines. (A) Total RNA was treated with RNase R. Hsa-circ-0007766 was stably expressed in both RNase R(-) and RNase $R(+)$ total RNA samples. (B) The divergent primers of hsa-circ-0007766 were designed, and the qRT-PCR product was verified with Sanger sequencing. (C) The result of Sanger sequencing, black arrow indicated the special splicing junction of hsa-circ-0007766. (D-E) qRT-PCR assay with divergent or 
convergent primers indicating the existence of hsa-circ-0007766 in GC cell lines. GAPDH was used as a negative control. (F) qRT-PCR assay with divergent primers indicated the high expression of hsa-circ-0007766 in 30 pairs of human GC tissues compared with their adjacent normal tissues. (G) The expression of hsa-circ-0007766 in SNU-216, SGC-7901, BGC-823, HGC-27, and GES-1 cell lines was measured by qRT-PCR. (H) qRT-PCR analysis verified the effective down-regulation of hsa-circ-0007766 after the transfection of the siRNA for 48 hours in SNU-216 or HGC-27 cells. (I) FISH detection of hsa-circ-0007766 in SNU-216 and HGC-27 cells. Nuclei were stained blue with DAPI. Hsa-circ- 0007766 was stained red with cy3. (Data are mean \pm SEM of three experiments. The Student's t-test analyzed the difference in F-H, $* P<0.05$ vs. Normal gastric tissues, GES-1, or negative control. Scale bar $=10 \mu \mathrm{m})$.

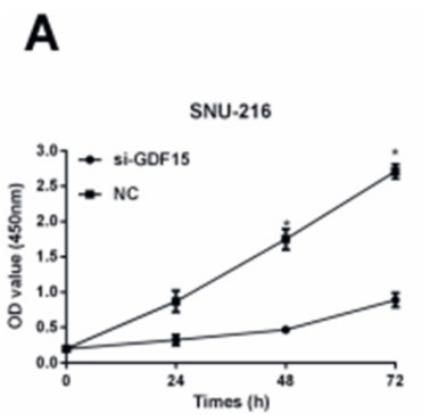

B

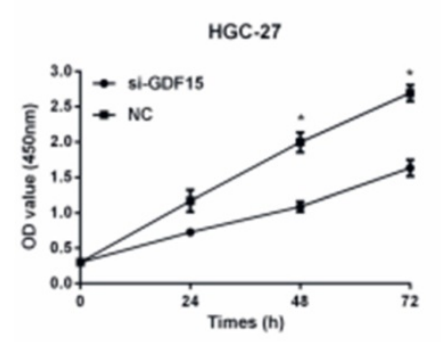

G

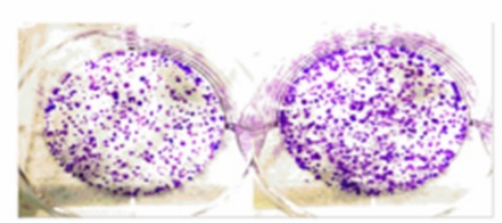

C

E

H
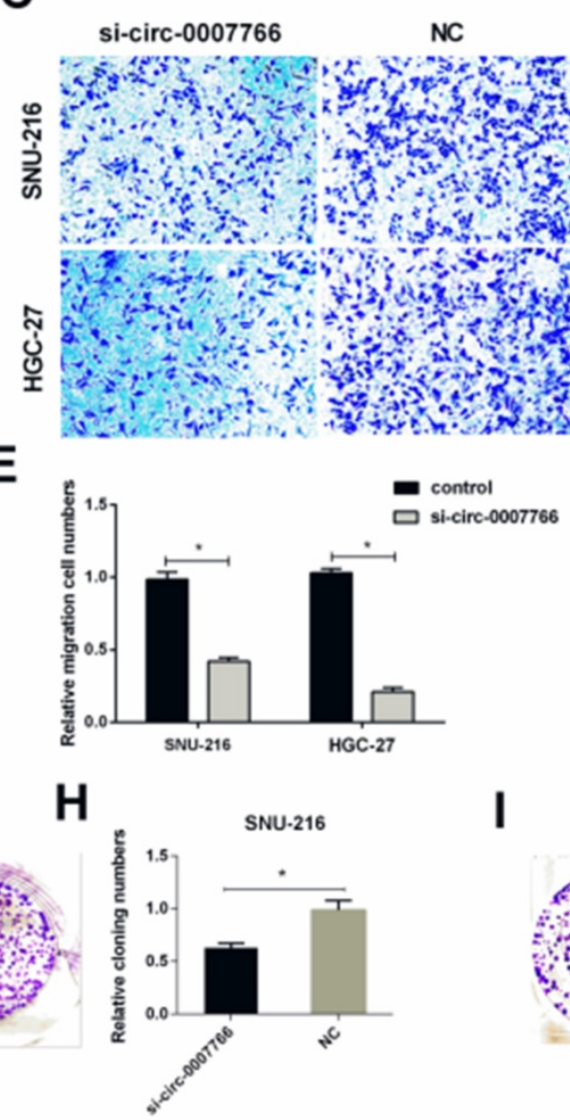

NC

I

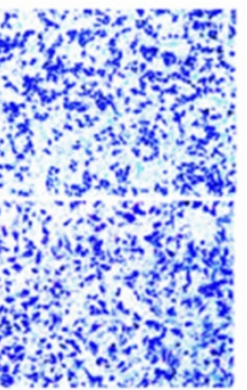

$\mathbf{F}$
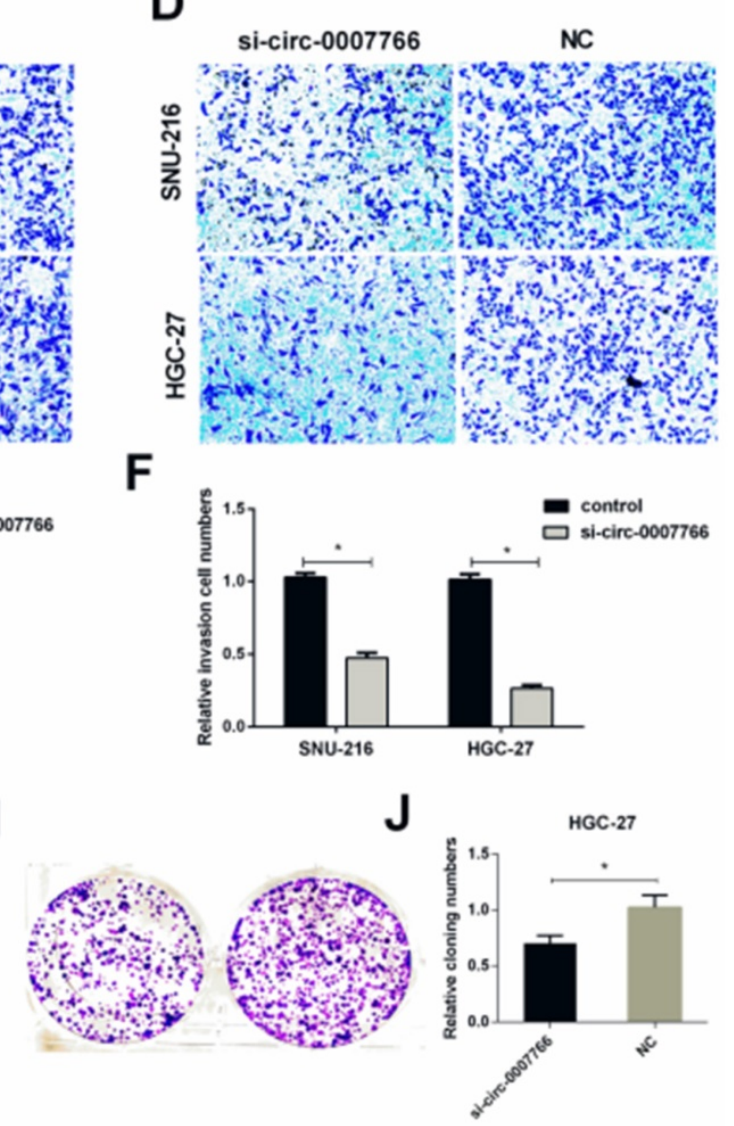

Figure 3. Down-regulated hsa-circ- 0007766 inhibited the progression of GC cells in vitro. (A) and (B) Down-regulation of hsa-circ-0007766 inhibited cell proliferation as indicated by CCK-8 assays in SNU-216 and HGC-27 cells. (C) and (E) Transwell migration assays were measured, and the results were expressed as relative migration cell numbers compared with respective control (magnification, $\mathrm{x} 100$, ${ }^{*} \mathrm{P}<0.05$, Student's t-test). (D) and (F) Transwell invasion assays were measured, and the results were expressed as relative invasion cell numbers compared with respective control (magnification, $x 100$, *P < 0.05, Student's t-test). (G-J) Down-regulation of hsa-circ-0007766 inhibited the colony formation of SNU-216 (G, H) and HGC-27 (I, J) cell lines as demonstrated by colony formation assays. (*P < 0.05 , Student's t-test).

\section{The silence of GDF15 inhibited the progression of $\mathbf{G C}$ cells in vitro}

We transfected GC cell lines SNU-216 and HGC-27 with siRNA for GDF15. Then, we assessed the effect of GDF15 on the proliferation, migration, and invasion of GC cells. qRT-PCR revealed that GDF15 was significantly down-regulated by GDF15 siRNA transfection compared with the negative siRNA control (Figure 6A). CCK-8 results showed that GDF15 knockdown dramatically reduced SNU-216 and HGC-27 cell proliferation compared with control groups (Figure 6B-6C). Knockdown of GDF15 also reduced the migration (Figure 6D-6E) and invasion (Figure 6F-6G) in SNU-216 and HGC-27 cells compared with cells transfected with the negative control. Next, we applied colony formation assays (Figure $6 \mathrm{H}$ ) to demonstrate that the down-regulation of GDF15 inhibited the colony formation of SNU-216
(Figure 6I) and HGC-27 (Figure 6J) cell lines. The correlation between GDF15 levels and the prognostic outcomes of GC patients was tested by the Kaplan-Meier Plotter website. Patients were stratified into two groups based on an auto select best cut-off. We revealed that GDF15 expression levels were negatively correlated with survival data (Figure 6K).

\section{MiR-1233-3p inhibitor (AMO-1233-3p) reversed the down-regulation effect of siRNA for hsa-circ-0007766 (si-circ) in GC cell lines}

To further verify the interaction between hsa-circ-0007766 and miR-1233-3p, and the binding between miR-1233-3p and GDF15, we used siRNA for hsa-circ-0007766 (si-circ) or si-circ plus miR-1233-3p inhibitor (AMO-1233 -3p) to transfect SNU-216 and HGC-27 cells. Subsequently, we used CCK-8 assay (Figure 7A-7B), transwell assay (Figure 7C-7F), and colony formation assay (Figure 7G-7H) to evaluate the 
effect of cell proliferation, migration, and invasion of GC cell lines SNU-216 and HGC-27. We found that AMO-1233-3p could significantly reverse the down-

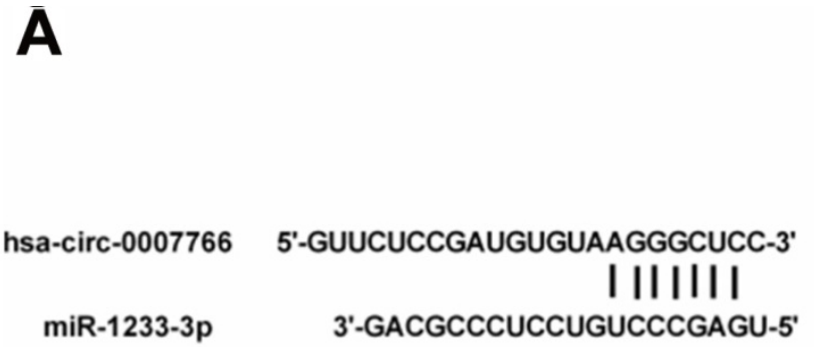

C

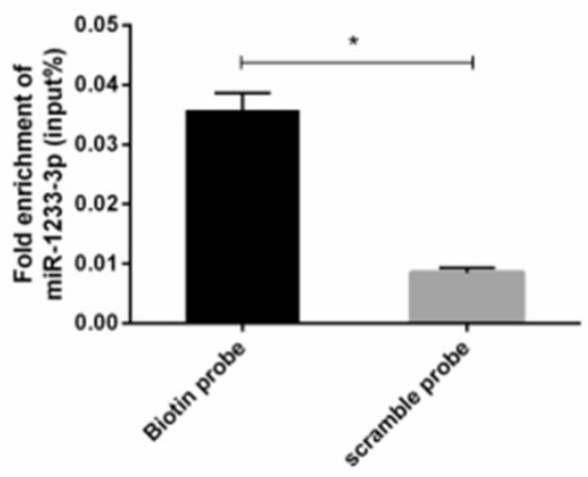

E

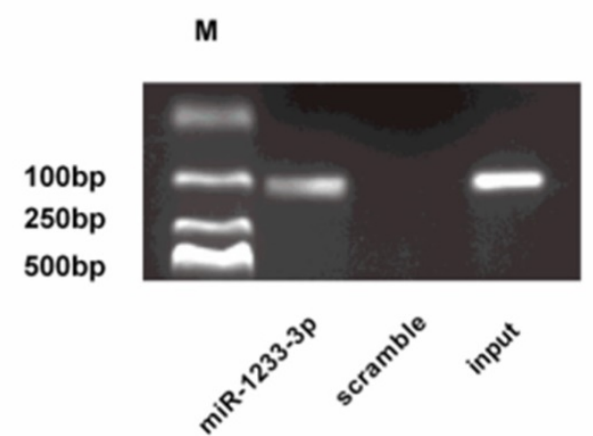

regulation effect of si-circ in GC cell lines SNU-216 and HGC-27.

B

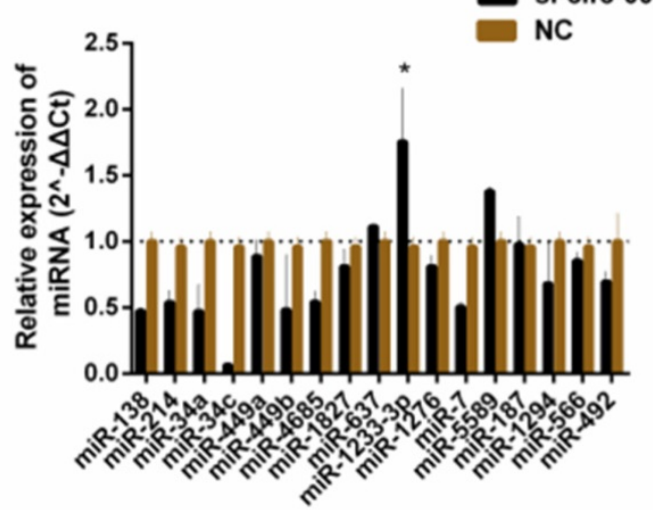

D

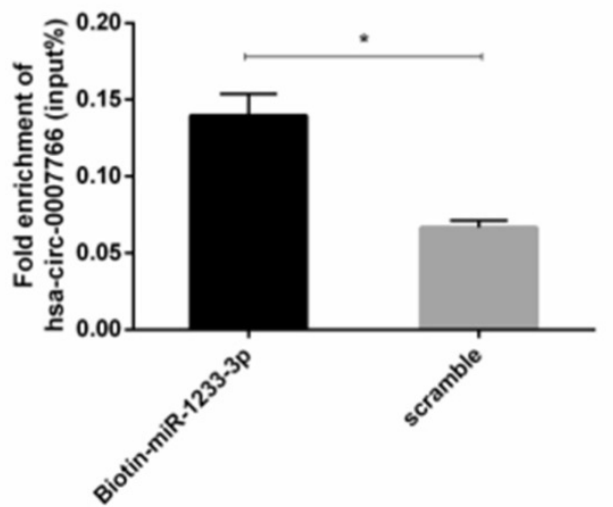

$\mathbf{F}$

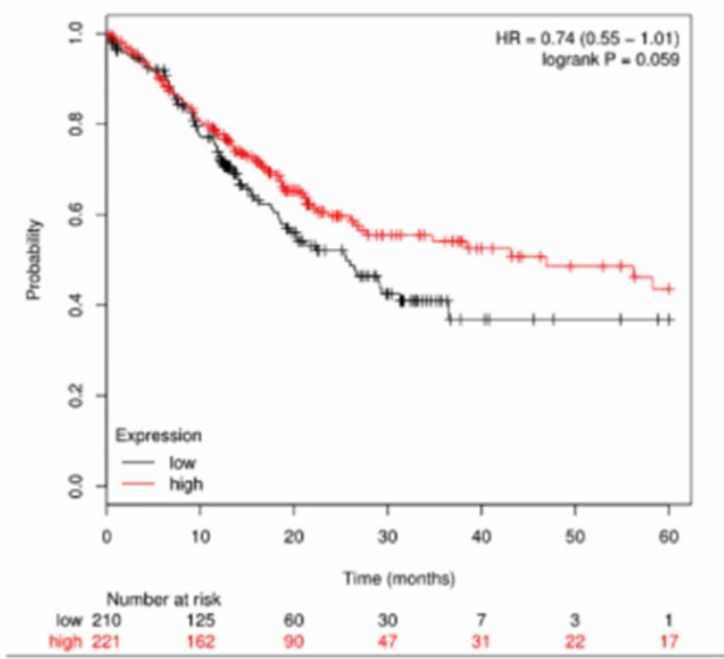

Figure 4. Hsa-circ-0007766 acted as a miRNA sponge for miR-1233-3p. (A) Bioinformatic analysis (Circlnteractome, Starbase V2.0, RNAhybrid, and TargetScan) of the putative binding sites of hsa-circ-0007766 with miR-1233-3p. (B) qRT-PCR assay with divergent primers verified the rise of miR-1233-3p after the transfection of siRNA for hsa-circ-0007766. (C) MiR-1233-3p pulled down by biotin-labelled hsa-circ-0007766 could be detected by qRT-PCR significantly compared with the scramble group. (D) Biotin-labelled miR-1233-3p captured more hsa-circ-0007766 than the scramble group. (E) The product of (D) was detected using qRT-PCR, followed by agarose gel electrophoresis. (F) Kaplan-Meier plotter analysis of the correlation between miR-1233-3p expression level and the overall survival of GC patients. (*P < 0.05, Student's t-test). 
A

$\begin{array}{cc}\begin{array}{c}\text { Position } 69-75 \text { of } \\ \text { GDF15-3'UTR }\end{array} & \text { 5'-CCUGCCCUGUGGAAUGGGCUCAA-3' }^{\text {IIIIII }} \\ \text { miR-1233-3p } & 3^{\prime} \text { '-GACGCCCUCCUGUCCCGAGU-5' }\end{array}$

C

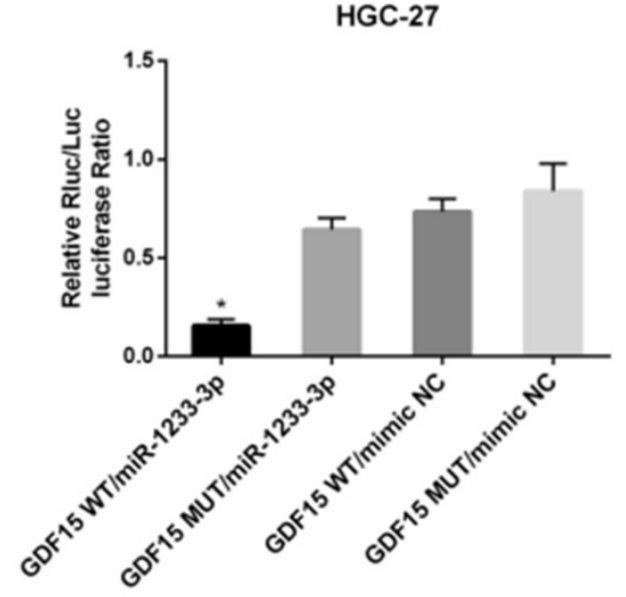

E

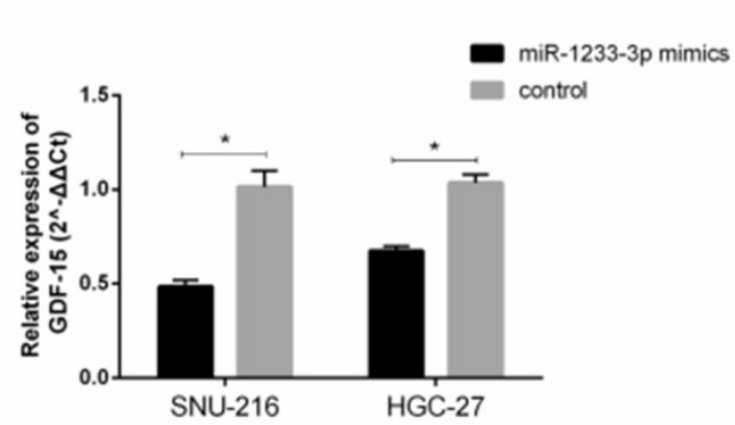

B

SNU-216

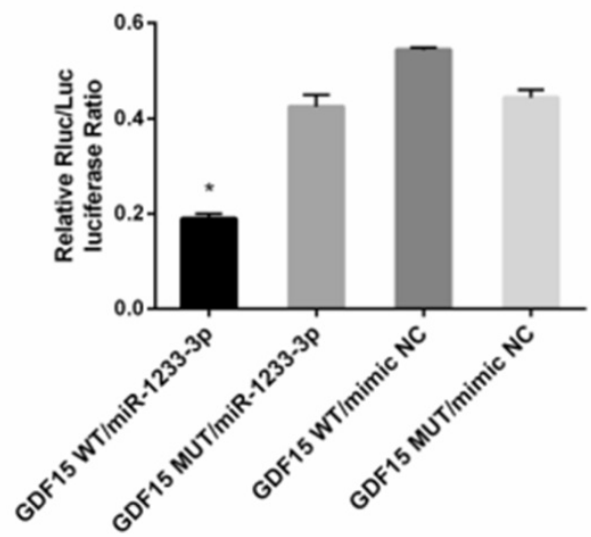

D

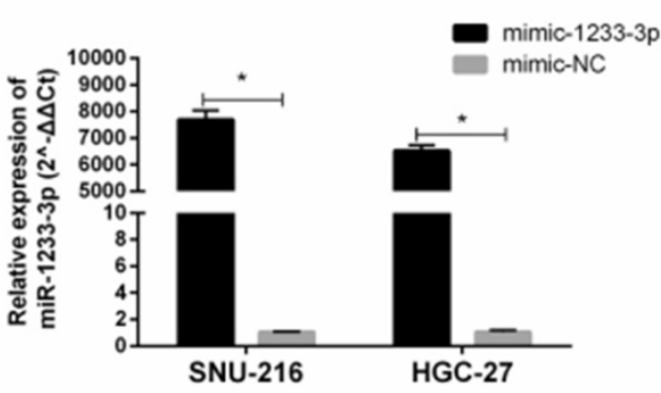

$\mathbf{F}$

SNU-216

HGC-27

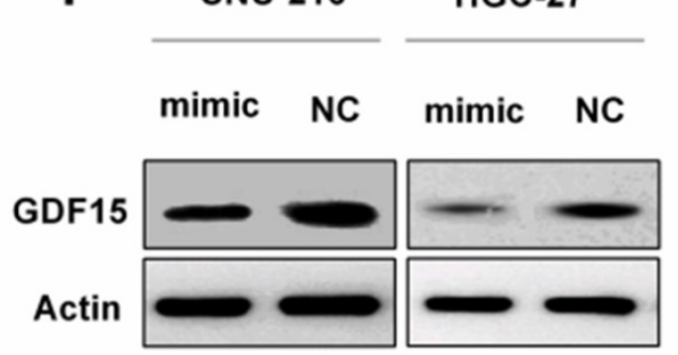

Figure 5. GDFI5 was a downstream target of miR-1233-3p. (A) Bioinformatic analysis (miRBase, Targetscan, RNAhybrid) of the putative binding sites of 3'UTR of GDFI5 with miR-1233-3p. (B) and (C) Dual-luciferase reporter assay was conducted to verify the binding between 3'UTR of GDFI5 (wild or mutant fragments) and miR-1233-3p in SNU-216 and HGC-27. We found that miR-1233-3p mimics could significantly reduce the ratios of luciferase activity. (D) MiR-1233-3p mimics could significantly up-regulate the expression of miR-1233-3p in SNU-216 and HGC-27. (E) Transfection of miR-1233-3p mimics down-regulated the expression of GDFI5 in SNU-216 and HGC-27. (F) Western blot assay showed that miR-1233-3p mimics could partly reduce the protein expression of GDFI5. (*P < 0.05 , Student's t-test).

\section{Hsa-circ-0007766 modulated the expression of miR-1233-3p target GDF 15}

Subsequently, we investigated whether hsacirc-0007766 exerted its effect by modulating the expression of GDF15. As shown in Figure 8A and 8D, the knockdown of hsa-circ-0007766 could down-regulate the expression of GDF15 by qRT-PCR or western blot. Besides, we co-transfected si-circ and AMO-1233-3p to detect the expression of GDF15 further. We found that the AMO-1233-3p could partially rescue the inhibitory effect of si-circ to the expression levels of GDF15 by qRT-PCR (Figure 8B) and Western blot (Figure $8 \mathrm{C}$ ). To investigate the underlying mechanisms of si-circ that suppressed cell proliferation, we measured a series of critical proteins related to cell progression by western blot assays. We found that GDF15, cell proliferation-related protein CyclinD1, and EMT-related protein Vimentin were down-regulated after the si-circ transfection compared with the negative control group (Figure 8D). 
A

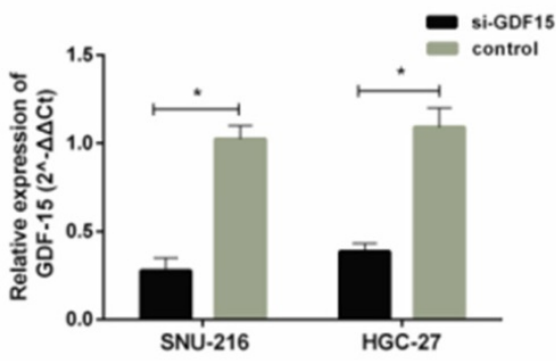

D

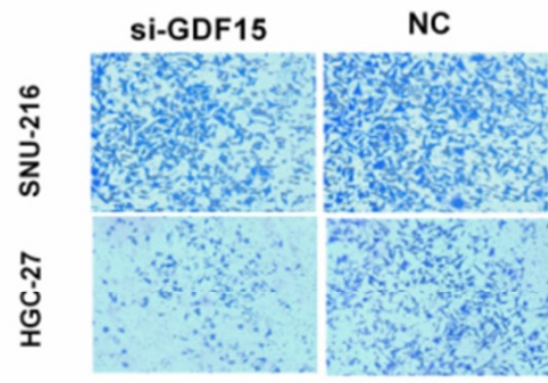

$\mathbf{F}$

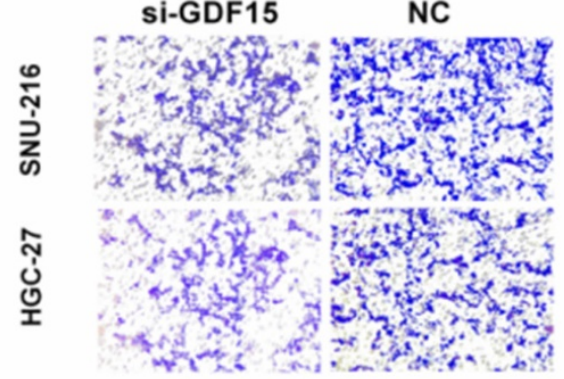

H

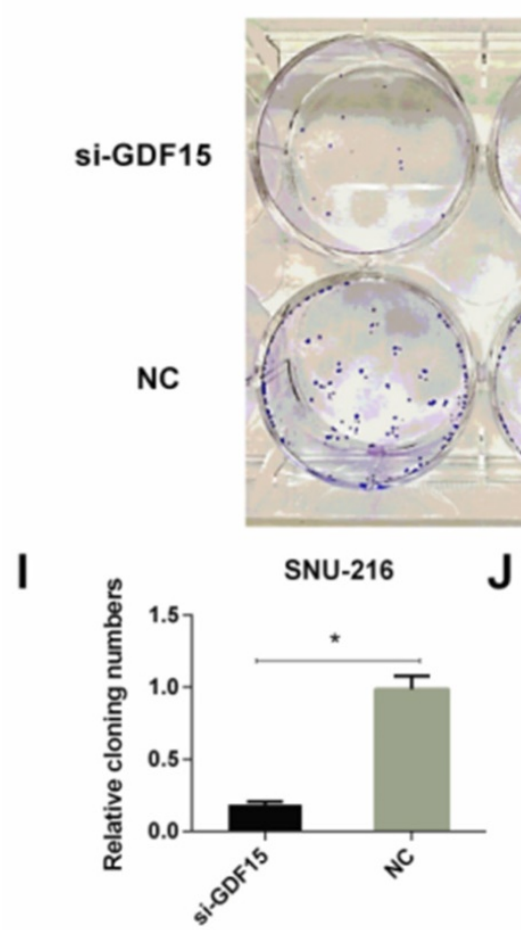

B

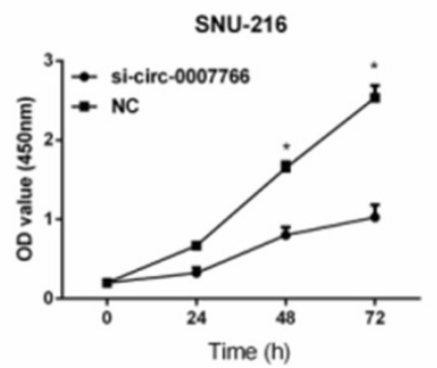

E

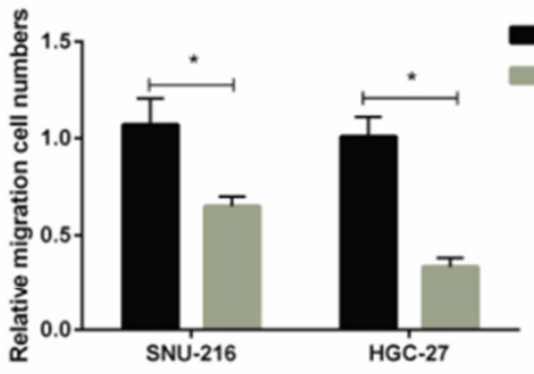

G

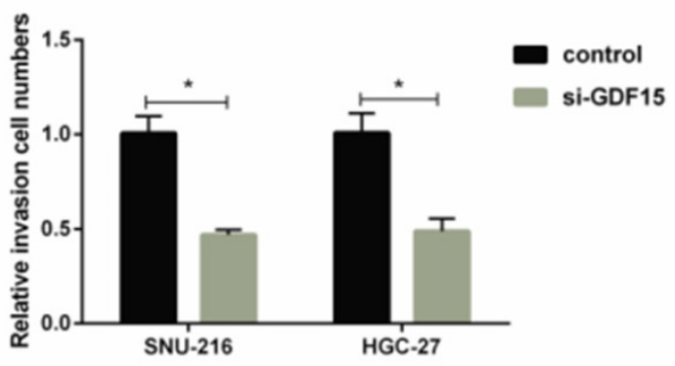

K

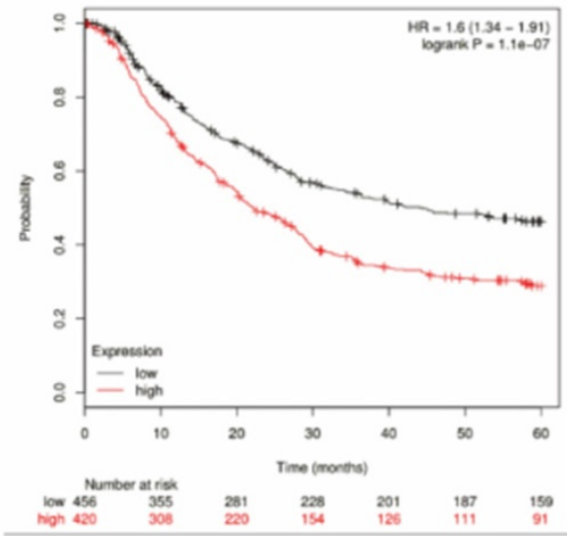

Figure 6. Silence of GDFI5 inhibited the progression of GC cells in vitro. (A) qRT-PCR analysis verified the effective down-regulation of GDFI5 after the transfection of the siRNA in SNU-216 or HGC-27 cells. (B) and (C) Silence of GDFI5 inhibited cell proliferation as indicated by CCK-8 assays in SNU-216 and HGC-27 cells. (D-G) Transwell migration (D-E) and invasion assays (F-G) were measured, and the results were expressed as relative migration and invasion cell numbers compared with respective 
control (magnification, x100, *P < 0.05, Student's t-test). (H-J) Down-regulation of GDFI5 inhibited the colony formation of SNU-216 (I) and HGC-27 (J) cell lines as demonstrated by colony formation assays. (K) Kaplan-Meier plotter analysis of the correlation between GDFI5 expression level and the overall survival of GC patients. (*P < 0.05 , Student's t-test).

A

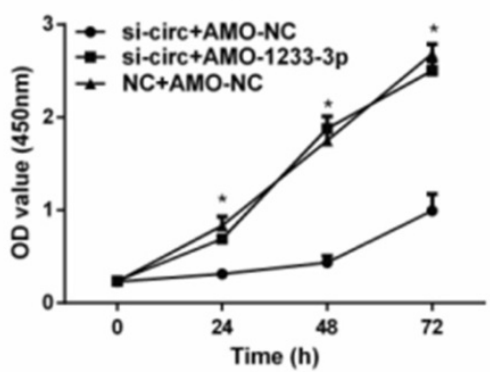

C

SNU-216

HGC-27

E

SNU-216

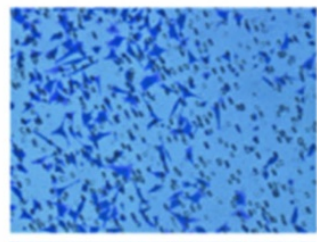

HGC-27

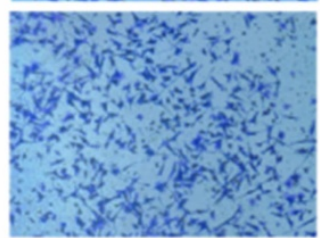

si-circ+

AMO-1233-3p
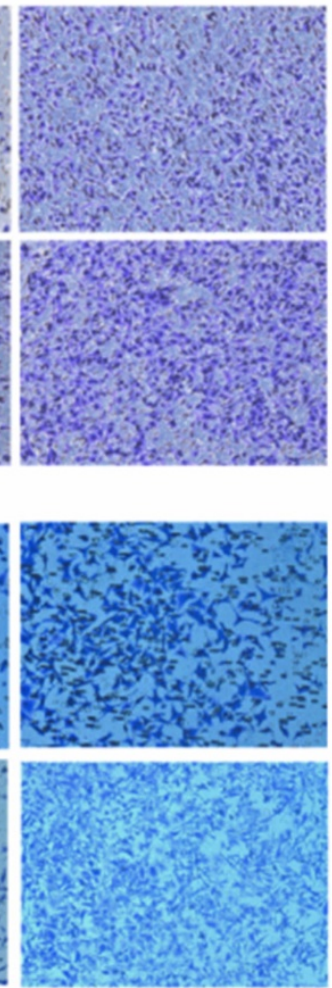

B

HGC-27

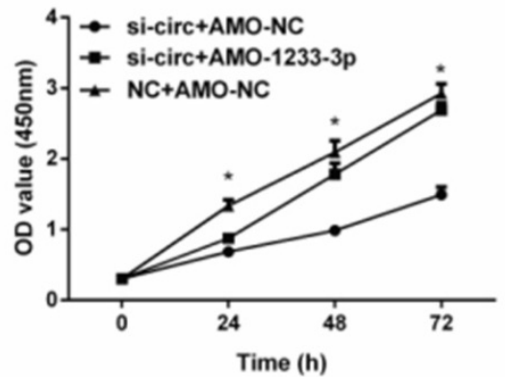

$\mathrm{NC}+$

AMO-NC

D
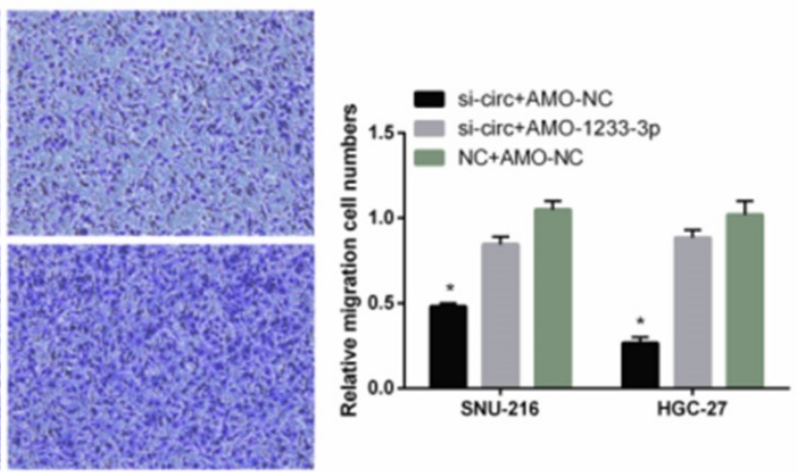

F
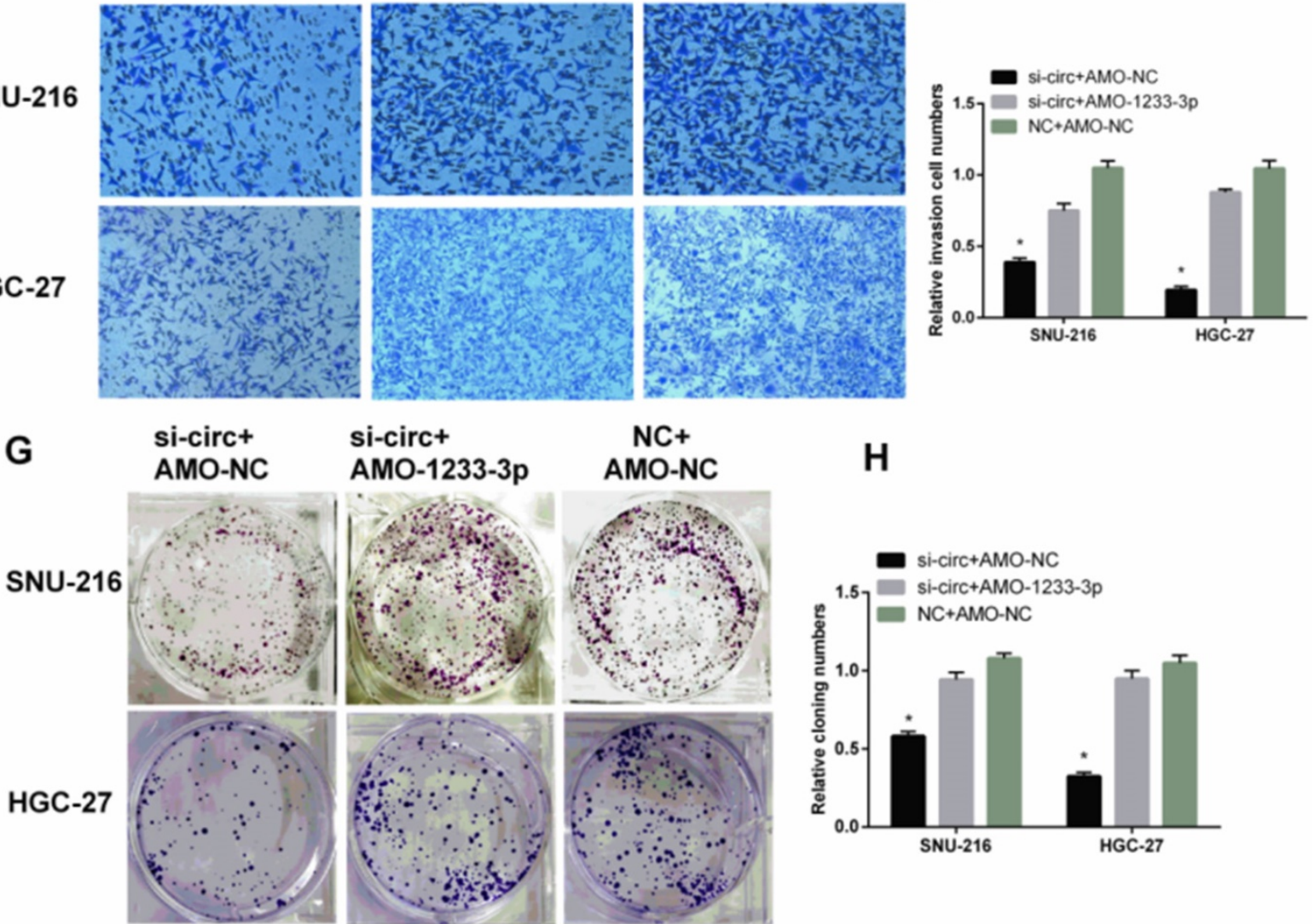

H

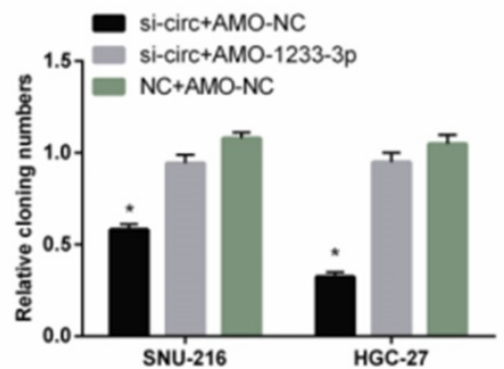

Figure 7. MiR-1233-3p inhibitor (AMO-1233-3p) reversed the down-regulation effect of siRNA for hsa-circ-0007766 (si-circ) in GC cell lines SNU-216 and HGC-27. (A) and (B) AMO-1233-3p reversed the inhibitory effect of si-circ on cell proliferation by CCK-8 assay. (C-F) AMO-1233-3p reversed the inhibitory effect of si-circ on cell migration (C-D) and invasion (E-F) by transwell assay. (G-H) Colony formation assay showed that the si-sirc+AMO-1233-3p group increased in colony numbers of SNU-216 and HGC-27 cell lines compared with the si-circ+AMO-NC group. (*P < 0.05, Student's t-test). 
A

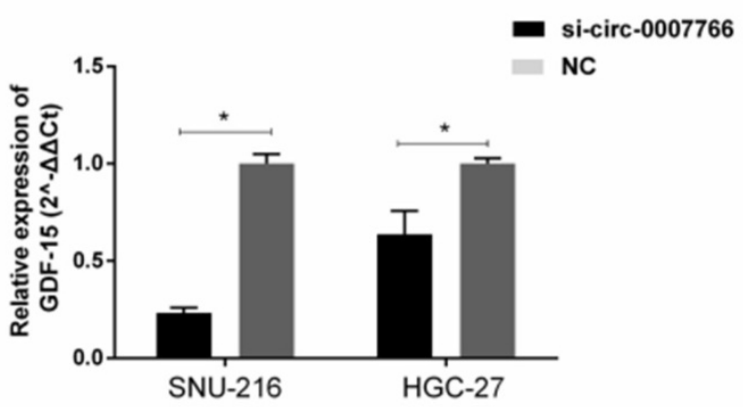

C

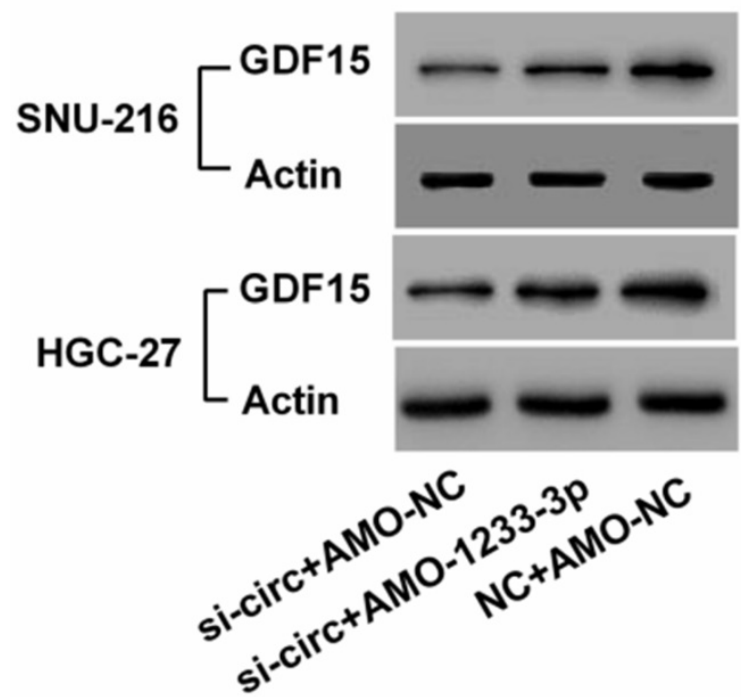

B

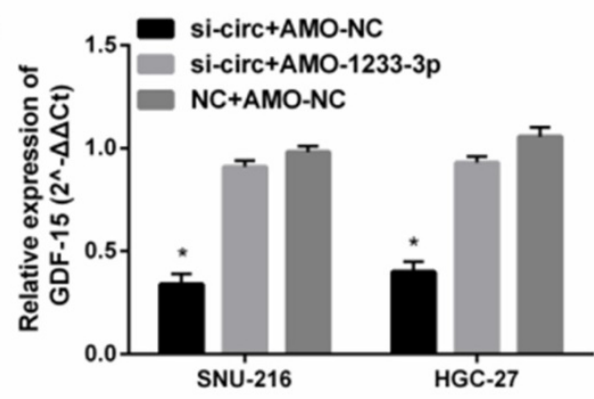

D

\section{GDF15}

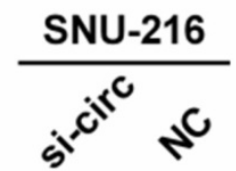

CyclinD1

Vimentin

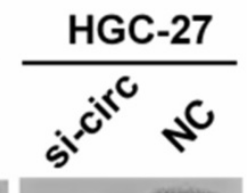

Actin
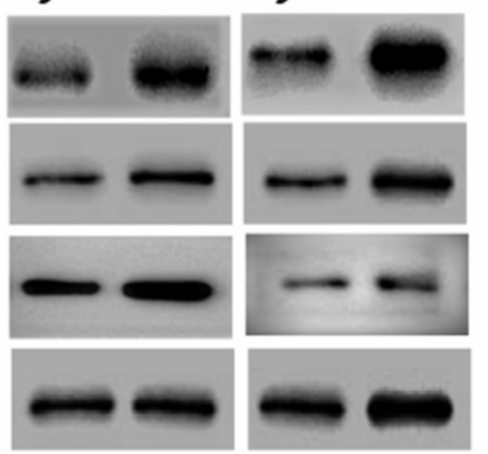

Figure 8. Hsa-circ-0007766 modulated the expression of miR-1233-3p target GDF15. (A) Transfection of siRNA for hsa-circ-0007766 inhibits the expression of GDF15. (B) The GDFI5 mRNA expression levels were reduced by the inhibition of hsa-circ-0007766 and reversed by the miR-1233-3p inhibitor (AMO-1233-3p). (C) The protein expression levels of GDF15 were in accordance with its mRNA expression levels. (D) Western blot analysis of GDFI5, cell proliferation-related protein CyclinDI, and EMT related protein Vimentin in GC cells transfected with siRNA for hsa-circ- 0007766 or negative control. ( $* \mathrm{P}<0.05$, Student's t-test).

\section{ROC analysis was conducted to evaluate the diagnostic value of hsa-circ-0007766 for GC patients}

Hsa-circ-0007766 was extracted from 30 pairs of gastric carcinoma tissues, and we differentiated tumors from matched adjacent normal tissues to evaluate the diagnostic value of hsa-circ-0007766 as a biomarker using ROC analysis. We found that the AUC was 0.704 (95\% CI, [0.572-0.815]; $\mathrm{P}=0.0024)$, with a cut-off value of 0.000709948 (sensitivity= $53.33 \%$; specificity $=83.33 \%$ ) (Figure 9).

\section{Discussion}

CircRNAs have been identified as abundant stable ncRNAs with high-throughput sequencing and bioinformatic analysis [10, 30]. However, in contrast to the full knowledge of lncRNAs and miRNAs, the function of circRNAs has been rarely elucidated.

Although studies have shown that the sponge function of circRNA is a kind of universal phenomenon $[5,6,8,31]$, some recent publications suggest that circRNAs do not necessarily function as miRNA sponges in human and mouse cells $[32,33]$. Compared with other competitive endogenous RNAs, circRNAs have more stable miRNA response elements and higher intracellular expression. Meanwhile, circRNAs are predominantly located in the cytoplasm and share the same location with miRNAs. However, a recent study also found circular intronic RNAs (ciRNAs) and EIciRNAs were mainly located in the nucleus and might act as the transcription regulators [34]. Furthermore, a few studies reported that some endogenous circRNAs 
with open reading frames or IRES could translate polypeptides or proteins under certain conditions $[7$, 13].

In this study, we found that hsa-circ-0007766 was highly expressed in gastric carcinoma tissues and verified its high expression in gastric carcinoma cell lines in vitro experiments. We then analyzed the results of qRT-PCR and found that the expression level of hsa-circ-0007766 was significantly correlated with histological grade. Next, we confirmed that convergent and divergent primers could both amplify cDNA of circRNA. However, gDNA could only be amplified by convergent primers. The results of Sanger sequencing verified the back-spliced junction, and the gel electrophoresis confirmed the product of qPCR for circRNA and linear RNA. Subsequently, we applied the technique of fluorescent in situ hybridization (FISH) to identify that hsa-circ-0007766 was mainly located in the cytoplasm. What's more, the CCK-8 assays, colony formation assays, transwell migration assays, and transwell invasion assays demonstrated that knockdown of hsa-circ-0007766 could significantly suppress the progression abilities of SNU-216 and HGC-27 cell lines. Taken together, these findings uncovered a remarkable role of hsacirc-0007766 as an oncogene in GC. Specially, we gradually confirmed the unique role of the hsacirc-0007766 in the gene regulatory network with pulldown assay and luciferase reporter assay, and the results were consistent with our hypothesis that hsacirc-0007766 regulates GDF15 by sponging miR-1233$3 p$. To our knowledge, this is the first report to illustrate the relation between circRNA and gene GDF15.

Bioinformatic analysis was applied to find the binding miRNAs with hsa-circ-0007766 (Figure 1). We searched CircInteractome, Starbase v2.0, Targetscan, and RNAhybrid, miR-1233-3p was finally picked out based on the context+ score percentile and PCR results of miRNAs from the intersection part of these databases [35-38]. Although the knockdown of certain circRNAs had been verified ineffective to the expression level of corresponding miRNAs by some studies $[5,8,31,39]$, we consistently discovered the stable and significant up-regulation of miR-1233-3p after the knockdown of hsa-circ-0007766 with siRNA. Then pulldown assay was conducted to confirm this binding effect [40]. It should be pointed out that several articles reported that circRNAs could mainly inhibit the activity rather than the transcription level of miRNAs to some extent $[5,39,41]$. Subsequently, we adopted the luciferase reporter assay to verify the binding site between miR-1233-3p and the 3'UTR of GDF15, which had been reported by Teng et al. [42]. We further confirmed the relation between GDF15 and $\mathrm{miR}-1233-3 p$ by regulating the miR-1233-3p with miRNA mimics or inhibitor to test the GDF15 mRNA and protein level via PCR and western-blot, respectively. The expression level of GDF15 was not significantly changed after the transfection of miR1233-3p inhibitor. This phenomenon might result from a low abundance of miR-1233-3p and a more prominent binding effect of other miRNAs.

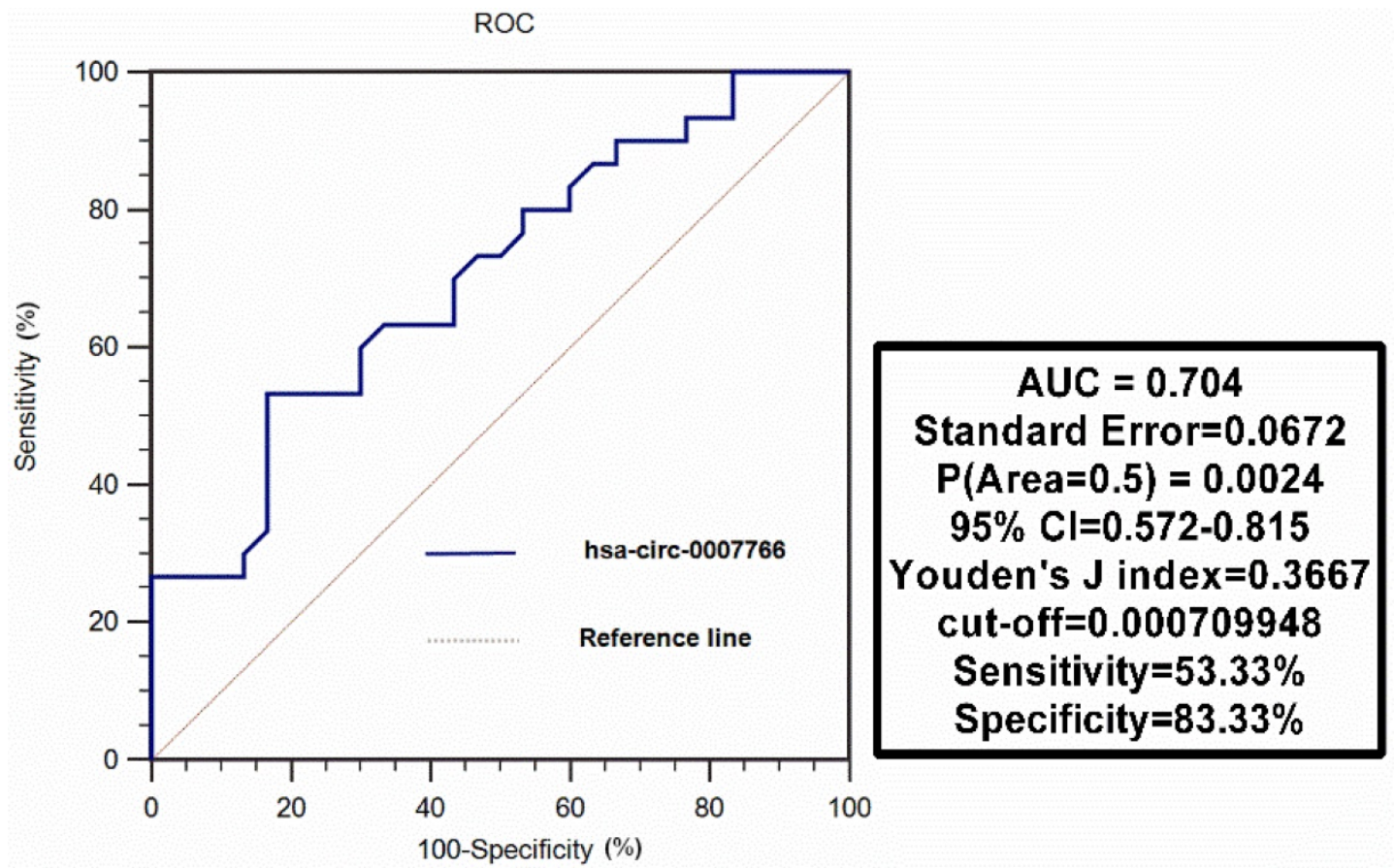

Figure 9. ROC curve analysis of hsa-circ-0007766 in gastric carcinoma tissues. Hsa-circ-0007766 was extracted from 30 pairs of gastric carcinoma tissues, and we evaluated the function of hsa-circ- 0007766 as a biomarker using ROC analysis. ROC: receiver operating characteristic, AUC: area under curve, Cl: confidence interval. 
Mir-1233-3p has been reported by several studies and may serve as a new biomarker for cancer diagnosis in the future $[41,43,44]$. This study further elaborated on the anti-tumor effect of miR-1233-3p, which was consistent with a previous report [41]. Some recent studies revealed that GDF15, also known as MIC-1, was related to the expression level of the iron regulatory protein hepcidin [45], cancer cachexia [21], and SMAD protein activation in the myocardium [23]. What's more, GDF15 could also act as an oncogene in GC as it might promote the progression of GC by stimulating GC cell invasion via an extracellular signal-regulated kinase-1/2-dependent pathway to up-regulate the uPA activation system [46]. Besides, several studies also elaborated on the characteristics of GDF15 to influence cancer stem-cell-like properties, activate apoptosis via the CXXC4-GDF15 axis, participates in fibroblast activation in GC [47]. Above all, GDF15 was widely accepted as a factor to promote cell viability, invasion, migration, angiogenesis, and apoptosis in human GC cell lines, which was consistent with our present research.

Finally, we conducted the ROC analysis and found that the AUC was 0.704 (95\% CI, [0.572-0.815]; $\mathrm{p}=0.0024$ ), with a sensitivity of $53.33 \%$ and a specificity of $83.33 \%$. The results indicated that the tissue level of hsa-circ-0007766 maybe as a potential diagnostic marker for GC.

In this study, we found that si-circ transfection impeded the expression of GDF15. Hence, we speculated that the knockdown of hsa-circ-0007766 could inhibit the progression of GC cell lines via the miR-1233-3p/GDF15 axis. Further studies are still needed to validate this mechanism. Moreover, accumulated evidence indicates that numerous circRNAs are enriched in the central neural system and not necessarily act as miRNAs sponge in human and mouse cell lines, so it should be further investigated concerning hsa-circ-0007766/miR-12333p/GDF15 axis in GC cell lines as the low abundance of hsa-circ-0007766. Furthermore, this axis may not play the dominant biological role in GC as the complexity of tumor heterogeneity.

In conclusion, our study explicitly elucidated the role of hsa-circ-0007766 in GC for the first time. The results showed that hsa-circ-0007766 was significantly high-expressed in GC and correlated with histological grade in terms of clinical-pathological parameters. Moreover, Down-regulated hsa-circ-0007766 suppressed cell proliferation, migration, and invasion in vitro. Subsequently, we explored the potential mechanism, and it suggested that hsa-circ-0007766 influenced the progression of GC cell lines via miR-1233-3p/GDF15 axis partly. Taken together, hsa- circ-0007766 could serve as a diagnostic and therapeutic biomarker for GC.

\section{Abbreviations}

ceRNA: competing endogenous RNA; circRNA: circular RNA; EIciRNAs: exon-intron circRNAs; miRNA: microRNA; GC: gastric carcinoma; GDF15: growth differentiation factor 15; qRT-PCR: quantitative real-time reverse transcriptionpolymerase chain reaction; CCK-8 assay: Cell Counting kit- 8 assay; ROC: receiver operating characteristic; AUC: area under curve; CI: confidence interval.

\section{Acknowledgments}

This work was supported by the Natural Science Foundation of China (grant number 81871718 and 81601843) and the Grand of Medicine innovation teams and Leading Talents of Jiangsu Health Department (CXTDA2017017). These funds covered publication costs.

\section{Competing Interests}

The authors have declared that no competing interest exists.

\section{References}

1. Hohenberger P, Gretschel S. Gastric cancer. Lancet. 2003; 362: 305-15.

2. Torre LA, Bray F, Siegel RL, Ferlay J, Lortet-Tieulent J, Jemal A. Global cancer statistics, 2012. CA Cancer J Clin. 2015; 65: 87-108.

3. Duarte HO, Gomes J, Machado JC, Reis CA. Gastric cancer: Basic aspects. Helicobacter. 2018; 23 Suppl 1: e12523.

4. Chen J, Li Y, Zheng Q, Bao C, He J, Chen B, et al. Circular RNA profile identifies circPVT1 as a proliferative factor and prognostic marker in gastric cancer. Cancer Letters. 2017; 388: 208-19.

5. Xie F, Li YW, Wang M, Huang C, Tao D, Zheng FX, et al. Circular RNA BCRC-3 suppresses bladder cancer proliferation through miR-182-5p/p27 axis. Molecular Cancer. 2018; 17.

6. Yang C, Yuan W, Yang X, Li P, Wang J, Han J, et al. Circular RNA circ-ITCH inhibits bladder cancer progression by sponging miR-17/miR-224 and regulating p21, PTEN expression. Molecular Cancer. 2018; 17.

7. Yang Y, Gao X, Zhang M, Yan S, Sun C, Xiao F, et al. Novel Role of FBXW7 Circular RNA in Repressing Glioma Tumorigenesis. Jnci-Journal of the National Cancer Institute. 2018; 110

8. Zheng Q, Bao C, Guo W, Li S, Chen J, Chen B, et al. Circular RNA profiling reveals an abundant circHIPK3 that regulates cell growth by sponging multiple miRNAs. Nature Communications. 2016; 7.

9. Chen L-L. The biogenesis and emerging roles of circular RNAs. Nature Reviews Molecular Cell Biology. 2016; 17: 205-11.

10. Qu S, Yang X, Li X, Wang J, Gao Y, Shang R, et al. Circular RNA: A new star of noncoding RNAs. Cancer Letters. 2015; 365: 141-8.

11. Rybak-Wolf A, Stottmeister C, Glazar P, Jens M, Pino N, Giusti S, et al. Circular RNAs in the Mammalian Brain Are Highly Abundant, Conserved, and Dynamically Expressed. Molecular Cell. 2015; 58: 870-85.

12. Li P, Chen S, Chen H, Mo X, Li T, Shao Y, et al. Using circular RNA as a novel type of biomarker in the screening of gastric cancer. Clinica Chimica Acta. 2015; 444: 132-6.

13. Legnini I, Di Timoteo G, Rossi F, Morlando M, Briganti F, Sthandier O, et al. Circ-ZNF609 Is a Circular RNA that Can Be Translated and Functions in Myogenesis. Molecular Cell. 2017; 66: 22-+.

14. Glažar P, Papavasileiou P, Rajewsky N. circBase: a database for circular RNAs. RNA (New York, NY). 2014; 20: 1666-70.

15. Dudekula DB, Panda AC, Grammatikakis I, De S, Abdelmohsen K, Gorospe M. CircInteractome: A web tool for exploring circular RNAs and their interacting proteins and microRNAs. RNA biology. 2016; 13: 34-42.

16. $\mathrm{Li} \mathrm{JH}$, Liu S, Zhou $\mathrm{H}, \mathrm{Qu}$ LH, Yang JH. starBase v2.0: decoding miRNA-ceRNA, miRNA-ncRNA and protein-RNA interaction networks from large-scale CLIP-Seq data. Nucleic acids research. 2014; 42: D92-7. 
17. Boku N. HER2-positive gastric cancer. Gastric cancer : official journal of the International Gastric Cancer Association and the Japanese Gastric Cancer Association. 2014; 17: 1-12.

18. Blanco-Calvo M, Tarrio N, Reboredo M, Haz-Conde M, Garcia J, Quindos M, et al. Circulating levels of GDF15, MMP7 and miR-200c as a poor prognostic signature in gastric cancer. Future Oncology. 2014; 10: 1187-202.

19. Ishige $T$, Nishimura $M$, Satoh M, Fujimoto M, Fukuyo M, Semba $T$, et al. Combined Secretomics and Transcriptomics Revealed Cancer-Derived GDF15 is Involved in Diffuse-Type Gastric Cancer Progression and Fibroblast Activation. Scientific reports. 2016; 6.

20. Kempf T, Zarbock A, Widera C, Butz S, Stadtmann A, Rossaint J, et al. GDF-15 is an inhibitor of leukocyte integrin activation required for survival after myocardial infarction in mice. Nature Medicine. 2011; 17: 581-U101.

21. Lerner L, Tao J, Liu Q, Nicoletti R, Feng B, Krieger B, et al. MAP3K11/GDF15 axis is a critical driver of cancer cachexia. Journal of Cachexia Sarcopenia and Muscle. 2016; 7: 467-82

22. Li C, Wang J, Kong J, Tang J, Wu Y, Xu E, et al. GDF15 promotes EMT and metastasis in colorectal cancer. Oncotarget. 2016; 7: 860-72.

23. Xu J, Kimball TR, Lorenz JN, Brown DA, Bauskin AR, Klevitsky R, et al. GDF15/MIC-1 functions as a protective and antihypertrophic factor released from the myocardium in association with SMAD protein activation. Circulation Research. 2006; 98: 342-50.

24. Lu L, Ma GQ, Liu XD, Sun RR, Wang Q, Liu M, et al. Correlation between GDF15, MMP7 and gastric cancer and its prognosis. European Review for Medical and Pharmacological Sciences. 2017; 21: 535-41.

25. Wang L, Liu Y, Li W, Song Z. Growth differentiation factor 15 promotes cell viability, invasion, migration, and angiogenesis in human liver carcinoma cell line HepG2. Clinics and Research in Hepatology and Gastroenterology. 2017; 41: 408-14.

26. Agarwal V, Bell GW, Nam J-W, Bartel DP. Predicting effective microRNA target sites in mammalian mRNAs. Elife. 2015; 4

27. Krüger J, Rehmsmeier M. RNAhybrid: microRNA target prediction easy, fast and flexible. Nucleic acids research. 2006; 34: W451-4.

28. Friedman RC, Farh KK, Burge CB, Bartel DP. Most mammalian mRNAs are conserved targets of microRNAs. Genome research. 2009; 19: 92-105.

29. Nagy A, Lanczky A, Menyhart O, Gyorffy B. Validation of miRNA prognostic power in hepatocellular carcinoma using expression data of independent datasets. Scientific reports. 2018; 8: 9227.

30. Zhang HD, Jiang LH, Sun DW, Hou JC, Ji ZL. CircRNA: a novel type of biomarker for cancer. Breast Cancer. 2018; 25: 1-7.

31. Xie $H$, Ren X, Xin S, Lan X, Lu G, Lin Y, et al. Emerging roles of circRNA_001569 targeting miR-145 in the proliferation and invasion of colorectal cancer. Oncotarget. 2016; 7: 26680-91.

32. Lasda E, Parker R. Circular RNAs: diversity of form and function. Rna. 2014; 20: $1829-42$

33. Memczak S, Jens M, Elefsinioti A, Torti F, Krueger J, Rybak A, et al. Circular RNAs are a large class of animal RNAs with regulatory potency. Nature. 2013; 495: 333-8.

34. Li Z, Huang C, Bao C, Chen L, Lin M, Wang X, et al. Exon-intron circular RNAs regulate transcription in the nucleus. Nature Structural \& Molecular Biology. 2015; 22: 256-64.

35. Dudekulay DB, Panda AC, Grammatikakis I, De S, Abdelmohsen K, Gorospe M. CircInteractome: A web tool for exploring circular RNAs and their interacting proteins and microRNAs. RNA biology. 2016; 13: 34-42.

36. Glazar P, Papavasileiou P, Rajewsky N. circBase: a database for circular RNAs. Rna-a Publication of the Rna Society. 2014; 20: 1666-70.

37. Karagkouni D, Paraskevopoulou MD, Chatzopoulos S, Vlachos IS, Tastsoglou $\mathrm{S}$, Kanellos I, et al. DIANA-TarBase v8: a decade-long collection of experimentally supported miRNA-gene interactions. Nucleic acids research. 2018; 46: D239-D45.

38. Li J-H, Liu S, Zhou H, Qu L-H, Yang J-H. starBase v2.0: decoding miRNA-ceRNA, miRNA-ncRNA and protein-RNA interaction networks from large-scale CLIP-Seq data. Nucleic acids research. 2014; 42: D92-D7.

39. Hansen TB, Jensen TI, Clausen BH, Bramsen JB, Finsen B, Damgaard CK, et al. Natural RNA circles function as efficient microRNA sponges. Nature. 2013; 495: 384-8.

40. Tan SM, Lieberman J. Capture and Identification of miRNA Targets by Biotin Pulldown and RNA-seq. Methods in molecular biology (Clifton, NJ). 2016; 1358: 211-28.

41. Zhang ZZ, Wang CJ, Niu L, Xu J, Wang M, Cao H, et al. Analysis of plasma MicroRNAs to identifying early diagnostic molecule for gastric cancer. International Journal of Clinical and Experimental Medicine. 2015; 8: 3700-U1798.

42. Teng M-S, Hsu L-A, Juan S-H, Lin W-C, Lee M-C, Su C-W, et al. A GDF15 3' UTR variant, rs1054564, results in allele-specific translational repression of GDF15 by hsa-miR-1233-3p. Plos One. 2017; 12

43. Wulfken LM, Moritz R, Ohlmann C, Holdenrieder S, Jung V, Becker F, et al. MicroRNAs in renal cell carcinoma: diagnostic implications of serum miR-1233 levels. PLoS One. 2011; 6: e25787.

44. Yadav S, Khandelwal M, Seth A, Saini AK, Dogra PN, Sharma A. Serum microRNA Expression Profiling: Potential Diagnostic Implications of a Panel of Serum microRNAs for Clear Cell Renal Cell Cancer. Urology. 2017; 104: 64-9.
45. Tanno T, Bhanu NV, Oneal PA, Goh SH, Staker P, Lee YT, et al. High levels of GDF15 in thalassemia suppress expression of the iron regulatory protein hepcidin. Nature Medicine. 2007; 13: 1096-101.

46. Lee DH, Yang Y, Lee SJ, Kim KY, Koo TH, Shin SM, et al. Macrophage inhibitory cytokine-1 induces the invasiveness of gastric cancer cells by up-regulating the urokinase-type plasminogen activator system. Cancer Res. 2003; 63: 4648-55.

47. Han M, Dai D, Yousafzai NA, Wang F, Wang H, Zhou Q, et al. CXXC4 activates apoptosis through up-regulating GDF15 in gastric cancer. Oncotarget. 2017; 8: 103557-67. 